\title{
Abordaje areolar en mamoplastia de aumento
}

\section{Areolar approach \\ in augmentation mammaplasty}

\author{
Castillo Delgado, P.*, Prado Scott, A.**, Troncoso Rigotti, A.***, \\ Villamán González, J .*, Fuentes Freire, P.*
}

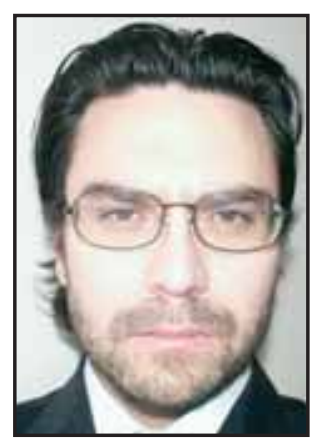

Castillo Delgado, $\mathbf{P}$.
Resumen

La mamoplastia de aumento se ha consolidado como una cirugía segura, de rápida recuperación y con resultados predecibles e inmediatos. Las expectativas de las pacientes son cada vez mayores, exigiendo resultados naturales y sin estigmas quirúrgicos. Todas las vías de abordaje clásicas, para la colocación de implantes mamarios, dejan cicatrices visibles. En mujeres con areolas de diámetro grande, es posible emplear un abordaje dentro de la areola, que deja una cicatriz oculta en su unión con el pezón. Se describe la técnica y posteriormente se discuten sus indicaciones, ventajas y desventajas.
Palabras clave Mamoplastia aumento. Prótesis mamarias

Código numérico 5521-5211402
Key words Breast augmentation. Breast implants

Numeral Code $\quad 5521-5211402$
Abstract

Augmentation mammoplasty has gained acceptance as a safe surgical procedure with fast recovery and a readily predictable outcome. Increasing patient expectations impose demands on natural appearance without signs of surgery. All standard incision areas for insertion of implant leave visible scars. For women with large areolar diameter, it is possible to leave a hidden scar at the aerola-nipple junction by conducting an intra- areolar approach. The technique is described and its intended application and advantages and disadvantages are discussed. 


\section{Introducción}

La mamoplastia de aumento es una de las cirugías cosméticas realizadas con mayor frecuencia en nuestro medio. Frente a cada paciente, el cirujano plástico debe considerar tres aspectos fundamentales: seleccionar el tipo y volumen del implante, definir la vía de abordaje y finalmente establecer el plano en que se instalará la prótesis $(1,2)$. Las vías de abordaje clásicas, incluyen la periareolar, submamaria y axilar, cada una de las cuales tienen ventajas y desventajas (3-6). Aunque con cualquiera de ellas es posible obtener excelentes resultados, todas dejan cicatrices visibles, que en algunos casos pueden perjudicar el resultado de la cirugía. En pacientes con areolas mayores de $4.5 \mathrm{~cm}$. de diámetro una alternativa es el abordaje descrito por Baxter, para la colocación de implantes salinos y que además permite reducir el diámetro areolar, sin dejar cicatrices visibles (7). Presentamos los casos de 3 pacientes, en las que hemos empleado este abordaje, utilizando implantes de gel de silicona.

Aunque este trabajo no presenta una técnica original, no hemos encontrado referencias de este abordaje en la literatura íbero-latinoamericana y por otra parte, lo hemos adaptado a implantes de gel de silicona, que son las prótesis mamarias empleadas con mayor frecuencia en nuestro medio.

\section{Material y método}

Empleamos el abordaje areolar en 3 pacientes de edades comprendidas entre los 25 y los 38 años, una de ellas con hijos y lactancias. Las pacientes fueron seleccionadas entre quienes consultaron por aumento mamario, con un diámetro areolar mayor de $4,5 \mathrm{~cm}$. y sin ptosis mamaria o que ésta fuera mínima, no requiriendo ningún procedimiento adicional para su corrección. El diámetro de las areolas varió en los tres casos presentados entre 4.5 y $6 \mathrm{~cm}$.

Utilizamos prótesis texturizadas de gel de silicona, de perfil alto, que fueron elegidas en conjunto con la paciente. Los volúmenes empleados estuvieron entre los 220 y 300 cc. Realizamos la cirugía con anestesia general y en decúbito dorsal, con las extremidades superiores abducidas en 90 grados. Se empleó profilaxis antibiótica, con Cefazolina.

La marcación específica de la técnica la realizamos con la paciente en decúbito dorsal e incluye el dibujo de 2 círculos concéntricos al pezón (Fig. 1). La marca interna se encuentra rodeando la base del pezón y la marca externa se localiza entre 1.5 a $2 \mathrm{~cm}$ del borde externo de la areola. Desepidermizamos la areola entre las dos marcas y a continuación realizamos una incisión en el tercio superior del área desepidermiza-

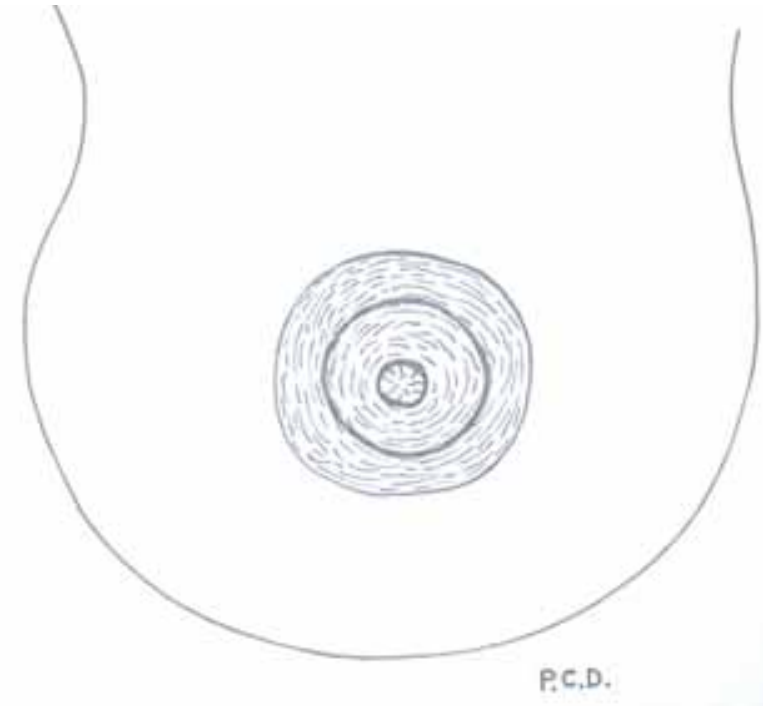

Fig. 1: Diseño cutáneo con dos círculos concéntricos al pezón. El interno en la base del pezón y el externo a 1,5-2 cm. Del borde areolar.

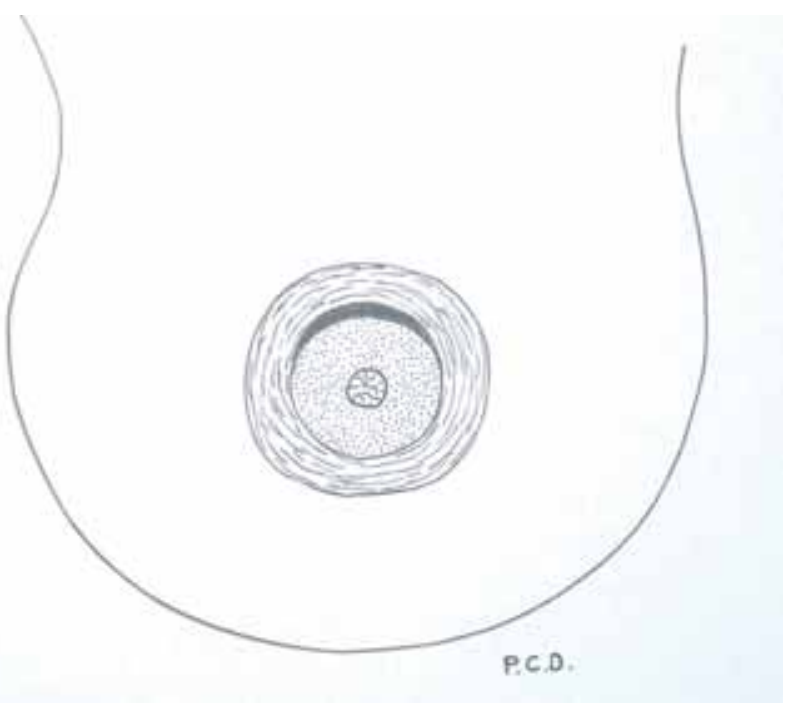

Fig. 2: Desepidermización del área marcada entre los dos círculos (en puntos). Acceso transglandular superior (en negro).

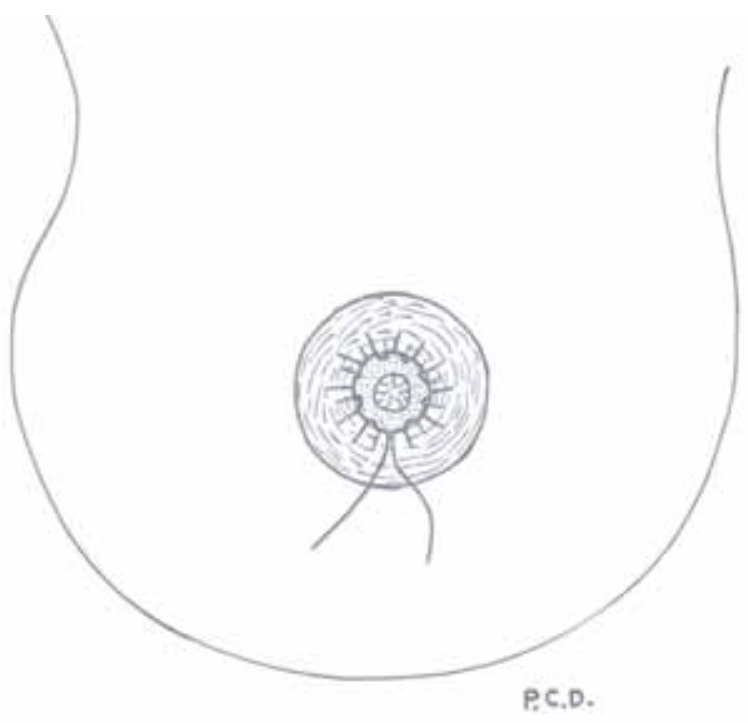

Fig. 3: Sutura en "round block" del borde areolar externo aproximándolo a la base del pezón. 
Tabla I. Puntuación escala Strasser

\begin{tabular}{lccc}
\hline & Paciente 1 & Paciente 2 & Paciente 3 \\
Malposición & 0 & 0 & 0 \\
Distorsión & 0 & 0 & 0 \\
Asimetría & 1 & 1 & 1 \\
Contorno & 0 & 0 & 1 \\
Cicatriz & 0 & 1 & 1 \\
TOTAL & $\mathbf{1}$ & $\mathbf{2}$ & $\mathbf{3}$ \\
\hline
\end{tabular}

Excelente 0; Bueno 1 a 4; Mediocre 5 a 14; Pobre más de 15

da (entre las 10 y 14 horas del reloj), que incide perpendicularmente la glándula accediendo al plano subfascial ( Fig. 2). Mediante un separador con luz disecamos el bolsillo, de acuerdo a las necesidades de cada paciente e implantamos la prótesis. Cerramos el parénquima con Poliglecaprone 3-0. Realizamos una sutura tipo "round block", con nylon 4-0 incoloro, aproximando el borde areolar desepidermizado, al pezón (Fig. 3). Finalizamos con puntos separados de nylon 6-0, que son retirados a la semana. No realizamos ningún manejo especial de la cicatriz y las pacientes fueron citadas regularmente a control.

Los resultados cosméticos y de la cicatrices fueron evaluados, a los 12 meses de evolución con las escalas de Strasser y de Beausang respectivamente $(8,9)$. Ambas escalas fueron aplicadas por un cirujano plástico ajeno al tratamiento de las pacientes y a la confección del trabajo. La sensibilidad del complejo areola pezón fue determinada subjetivamente por la propia pacientes.

\section{Resultados}

El acceso areolar nos permitió disecar el bolsillo deseado e instalar la prótesis sin dificultad. El tiempo quirúrgico fluctuó entre 2 y 3 horas y el seguimiento ha sido de entre 12 y 18 meses. No hubo hematomas u otras complicaciones quirúrgicas.
Tabla II. Puntuación escala Beausang

\begin{tabular}{lccc}
\hline & Paciente 1 & Paciente 2 & Paciente 3 \\
Color & 1 & 2 & 2 \\
Mate & 1 & 1 & 1 \\
Contorno & 1 & 1 & 1 \\
Distorsión & 1 & 1 & 1 \\
Textura & 1 & 1 & 1 \\
Escala visual & 0 & 0 & 0 \\
TOTAL & $\mathbf{5}$ & $\mathbf{6}$ & $\mathbf{6}$ \\
\hline
\end{tabular}

De acuerdo a la escala de Strasser, las pacientes tienen una puntuación de 1,2 y 3 puntos respectivamente, correspondiendo a buenos resultados (Tabla I). Las cicatrices evaluadas según Beausang tuvieron una puntuación de 5, 6 y 6 puntos respectivamente, evidenciándose sólo una discreta decoloración, en las dos últimas pacientes (Tabla II). Las cicatrices han permaneciendo ocultas en la unión areola-pezón y de acuerdo a la escala de Beausang, se encuentran en un rango excelente. Durante el primer mes se observaron algunos pliegues cutáneos radiados alrededor del pezón, que desaparecieron espontáneamente. Consultadas específicamente por la sensibilidad del complejo areola pezón, ninguna paciente refirió alteraciones sensitivas.

En ningún caso se ha evidenciado contractura capsular y todas las pacientes han manifestado un alto grado de satisfacción, especialmente por no tener cicatrices visibles. Aunque uno de los objetivos de la cirugía era reducir el diámetro de las areolas, este cambio no fue muy significativo, evidenciándose sólo una disminución de entre 4 y 8 mm (Fig. 4-6).

\section{Discusión}

La mamoplastia de aumento es una cirugía en que el resultado es inmediatamente evidente, dejando habitualmente satisfechos a pacientes y cirujanos. Sin embargo, las pacientes cada vez se encuentran mejor

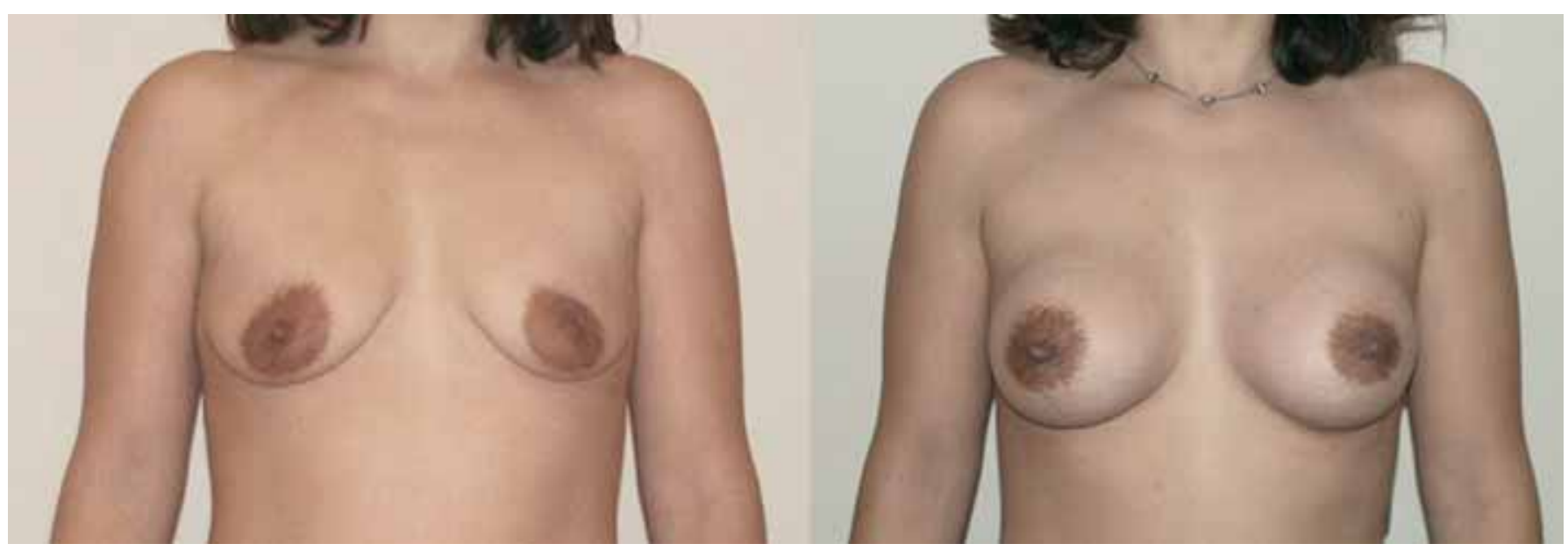

Fig. 4: Resultados de mamoplastia de aumento con abordaje areolar: prótesis redondas de perfil muy alto de $220 \mathrm{cc}$. 


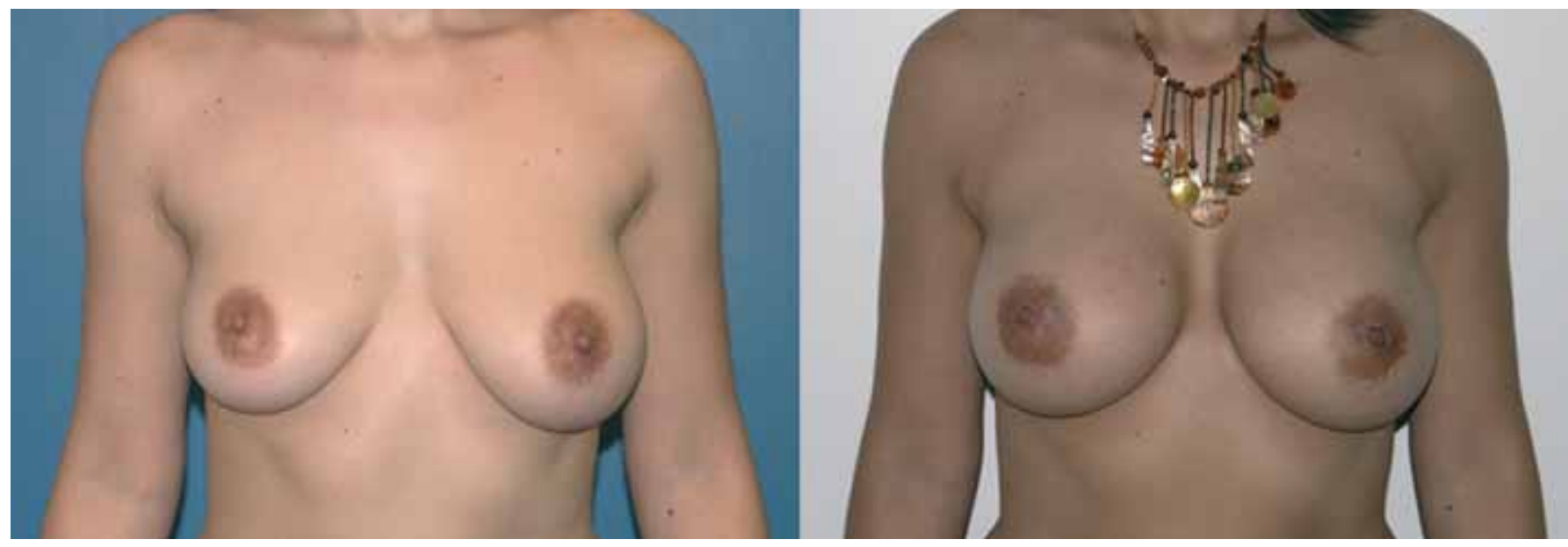

Fig. 5: Resultados de mamoplastia de aumento con abordaje areolar: prótesis redondas de perfil muy alto de 260 cc.

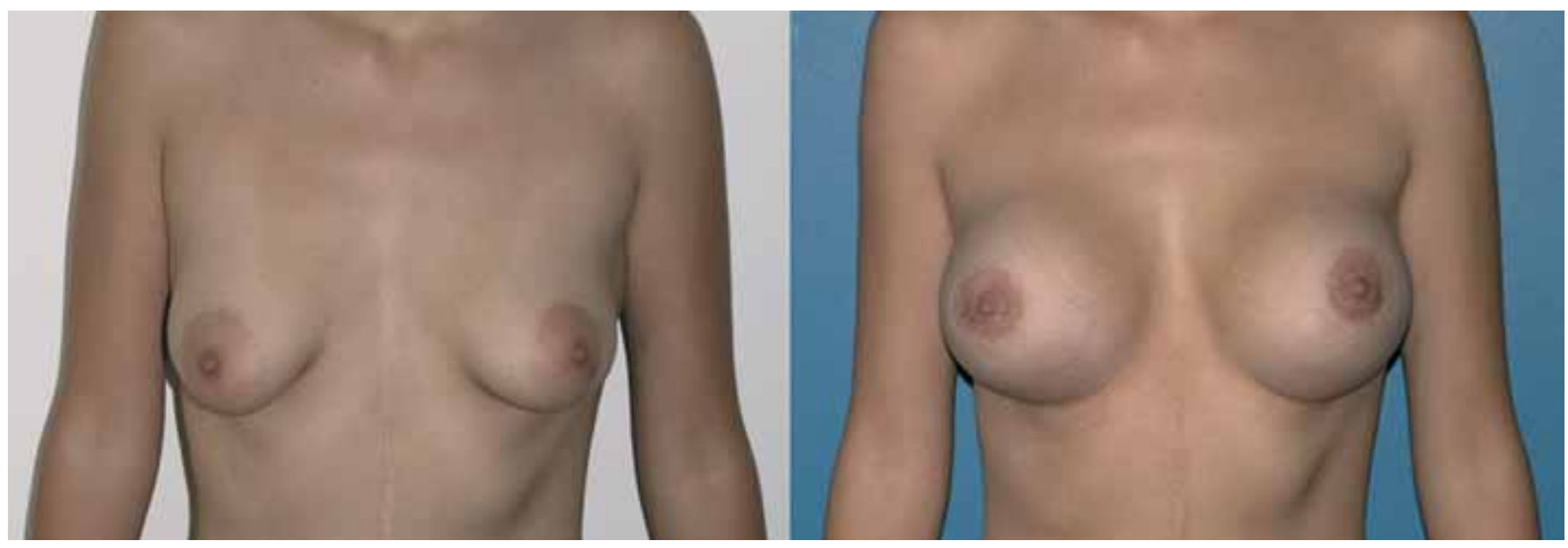

Fig. 6: Resultados de mamoplastia de aumento con abordaje areolar: prótesis redondas de perfil muy alto de $300 \mathrm{cc}$.

informadas y con mayores expectativas. Una de las preocupaciones habituales es la localización y tamaño de la cicatriz. Aunque con cualquiera de los abordajes clásicos, submamario, periareolar o axilar, es posible obtener excelentes resultados, todos dejan una cicatriz visible. Ocasionalmente esta cicatriz puede ensancharse, sufrir alteraciones en su pigmentación o presentar alteraciones hipertróficas, perjudicando el resultado cosmético de la cirugía.

Con el abordaje areolar descrito es posible acceder a cualquiera de los planos en que se colocan los implantes mamarios, ya sea subglandular, subfascial o submuscular. Su principal ventaja es que no deja cicatriz visible, ya que ésta queda oculta entre la areola y la base del pezón. Aunque en 2 de nuestras pacientes se produjo una discreta decoloración alrededor del pezón, esta no ha representado una alteración cosmética significativa (Fig. 7).

La técnica que presentamos fue descrita por Baxter para mamoplastias de aumento con implantes salinos alojados en el plano submuscular (7). En nuestro medio los implantes utilizados con mayor frecuencia son los de gel de silicona, que han demostrado su

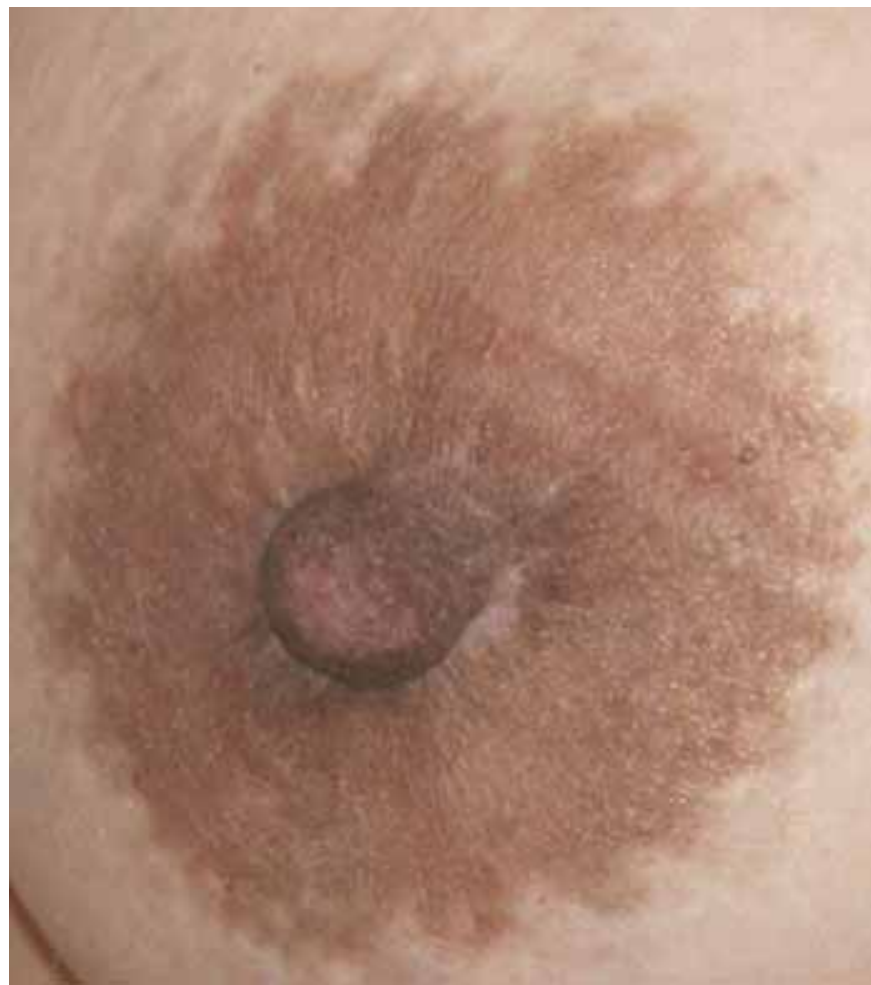

Fig. 7: Acercamiento de la cicatriz en mama derecha evidenciándose sólo discreta decoloración en la unión de areola y pezón. 
seguridad, habiéndose descartado su asociación con cáncer de mama o enfermedades autoinmunes (1014). Aunque introducir una prótesis texturizada de gel de silicona por esta vía, puede ser técnicamente más complejo, en todas nuestras pacientes fue posible emplear el implante deseado.

Esta técnica requiere de areolas mayores de 4.5 $\mathrm{cm}$. de diámetro, en las que además resulte estéticamente beneficioso reducir su diámetro. Una desventaja del acceso areolar, al igual que en la mayoría de los abordajes periareolares, es la necesidad de cortar el parénquemia mamario, con el riesgo potencial de colonizar la prótesis con Estafilococo epidermidis. Empleamos profilaxis antibiótica en las tres pacientes y no hemos tenido casos de infección y aunque el número de pacientes es limitado y el tiempo de seguimiento es escaso, tampoco hemos evidenciado contractura capsular. En las 3 pacientes hemos empleado un abordaje areolar superior, sin embargo, también existe la posibilidad de realizar un abordaje areolar inferior (equivalente a un periareolar inferior), lo cual depende de la experiencia y preferencias del cirujano. Aunque no hemos empleado este último, creemos que no debieran existir mayores diferencias en la técnica o en los resultados.

Uno de los objetivos de la técnica es reducir el diámetro de las areolas, sin embargo al evaluar los resultados, evidenciamos que a pesar de la importante resección de piel areolar, la areola no redujo significativamente su tamaño. Esto lo atribuimos a que al instalar el implante, se produce una distensión cutánea de la mama y la areola que determina su dilatación. Sin embargo, al aumentar globalmente de volumen la mama, la areola proporcionalmente se ve más pequeña y armónica, por lo que a ninguna de las pacientes de nuestro estudio le llamó la atención este hecho.

La técnica está especialmente indicada en pacientes con hipoplasia mamaria y areolas de diámetro grande, en las que se plantea simultáneamente su reducción. Además las mamas no deben presentar ptosis o ésta debiera ser mínima, no justificando ningún tipo de procedimiento adicional para su corrección.

Hemos empleado esta técnica en 3 casos con seguimiento de entre 12 y 18 meses, obteniendo un alto nivel de satisfacción por parte de las pacientes. Aunque nuestra casuística es pequeña, hemos querido dar a conocer estos casos para ayudar a difundir una técnica poco conocida y que estando bien indicada puede ser una valiosa alternativa.

\section{Conclusiones}

El abordaje areolar es un acceso poco conocido para mamoplastias de aumento y que debiera tenerse presente, ya que tiene la ventaja de dejar una cicatriz oculta en la unión de la areola con el pezón, minimizando los estigmas quirúrgicos. Requiere de una adecuada selección de las pacientes, que deben tener un diámetro areolar mayor de 4,5 c. y no deben presentar ptosis mamaria o ésta debe ser mínima. A través de esta vía pueden colocarse implantes en cualquiera de los planos, ya se subglandular, subfascial o submuscular.

\section{Dirección del autor}

Dr. Paulo Castillo Delgado.

Santa Marta 7650. Comuna de Huechuraba.Santiago (Chile)

e-mail: castilloplast@gmail.com

\section{Bibliografía}

1. Spear S, Bulan E, Venturi M.: “ Breast augmentation”. Plast. Reconst. Surg. 2004; 114(5): 73.

2. Brody G.: "Breast implant size selection and patient satisfaction". Plast. Reconst. Surg. 1981; 68(4): 611.

3. Hidalgo D.: "Breast augmentation: Choosing the optimal incision, implant, and pocket plane". Plast. Reconstr. Surg. 2000; 105(6): 2202.

4. Hoehler H.: "Breast augmentation: The axillary approach". Br J Plast Surg 1973: 26: 373.

5. Biggs T, Cukier J, Worthing L.: "Augmentation mammaplasty: a review of 18 years". Plast. Reconst. Surg. 1982; 69(3): 445.

6. Graf R, Bernardes A, Rippel R, Araujo L Damasio R Auersvald A.: "Subfascial breast implant: a new procedure". Plast. Reconst. Surg. 2003; 111(2): 904.

7. Baxter R.: "Nipple or areolar reduction with simultaneous breast augmentation". Plast. Reconst. Surg. 2003; 112(7): 1918.

8. Strasser, E. J.: "Application of an objective grading system for the evaluation of cosmetic surgical results". Plast. Reconstr. Surg. 109(5): 1733.

9. Beausang, E., Floyd, H., Dunn, K. W., Orton, C. I., and Ferguson, M. W.: "A new quantitative scale for clinical scar assessment". Plast. Reconstr. Surg. 1998, 102(6): 1954.

10. Muzaffer A, Rohrich R.: "The silicone gel-filled breast implant controversy: An update”. Plast. Reconstr. Surg. 2001; 109(2): 742.

11. Angell M.: "Evaluating the health risks of breast implants: The interplay of medical science, the law, and public opinion". N. Engl. J. Med. 1996; 334(23): 1513.

12. Deapen D. Pike M. Casagrande J.: "The relationship between breast cancer and augmentation mammaplasty: An epidemiologic study". Plast. Reconstr. Surg. 1986; 77(3): 361.

13. Rohrich R. Hollier L. Robinson J.: "Determining the safety of the silicone envelope: In search of a silicone antibody". Plast. Reconstr. Surg. 1996; 98(3): 455.

14. Su C, Dreyfuss D, Krizek T.: "Silicone implants and the inhibition of cancer". Plast. Reconstr. Surg. 1995; 96(3): 513. 


\section{Comentario al trabajo uAbordaje areolar en mamoplastia de aumento॥}

\section{Dt. Thomas M. Biggs. Cirujano Plástico. Director de la Revista Aesthetic Plastic Surgery. Houston, Texas. EEUU.}

He leído atentamente el Trabajo del Dr. Castillo Delgado y examinado las fotografías que lo ilustran. La utilización de la vía circumareolar en la mamoplastia de aumento no es infrecuente. Benelli y Sampaio Goes la han estado empleando durante casi 20 años y otros muchos autores también han empleado un abordaje circum-vertical tanto en mastopexia como en mamoplastia de reducción (Graf, Biggs, Motura, et al.)El trabajo del Dr. Castillo Delgado tiene como mérito el confirmar el dato de que se puede al mismo tiempo tener un buen acceso al espacio deseado, reducir la circunferencia de la areola y no dejar cicatrices inoportunas. Es también de remarcar el hecho de que no compruebe una reducción significativa en el diámetro areolar en sus tres pacientes; intuyo que puede ser debido a una tensión inadecuada en la sutura en "round block". En mi experiencia trabajando con Benelli (y con Goes) en numerosas ocasiones, he seguido sus instrucciones y siempre he obtenido una reducción en el diámetro de la areola cuando éste era el objetivo. Animo al Dr. Castillo a seguir su trabajo en este campo.

\section{Respuesta al comentario del Dr. Thomas M. Biggs}

\section{Dr. Castillo Delgado, $\mathbf{P}$.}

Agradezco el comentario del Dr. Biggs, cirujano plástico de amplia experiencia en cirugía mamaria. A diferencia del procedimiento de Benelli, al cual hace referencia el Dr. Biggs y que deja una cicatriz periareolar; en este trabajo se presenta una técnica en que la resección cutánea se realiza al centro de la areola, dejando una cicatriz en unión de la areola con el pezón y no periareolar, lo que la hace virtualmente imperceptible. Para evitar tensión en el cierre, la cantidad de piel areolar que puede eliminarse, es significativamente menor que en la técnica de Benelli. Esto determinaría una reducción limitada del diámetro areolar. Sin embargo, al aumentar la mama globalmente su volumen, la areola aparece proporcionalmente más pequeña, por lo que ninguna de las pacientes presentó algún reparo. 